\title{
Cambios esperados en relaciones interpersonales después de emergencia sanitaria. Delphi entre directivos españoles y colombianos
}

\section{Expected changes in interpersonal relationships after health emergency. Delphi analysis among managers in Spain and Colombia}

Artículo de investigación científica y tecnológica.

Fecha de recepción: 29/09/2020

Fecha de devolución: 01/03/2021

Fecha de aceptación: 22/03/2021

Fecha de publicación: 26/03/2021

\section{Marcela Navia-Núñez Infocaribe / CEIPA}

Barranquilla, Atlántico (Colombia) contacto@infocaribe.info

\section{Yanna Stefanu- Lidorikiotu \\ Universidad de La Rioja / \\ Universidad de Barcelona \\ Logroño, La Rioja (España) \\ yanna.stefanu@gmail.com}

Para citar este artículo:

Navia-Núñez, M. \& Stefanu-Lidorikiotu, Y. (2021). Cambios esperados en relaciones interpersonales después de emergencia sanitaria: Delphi entre directivos españoles y colombianos. Económicas CUC, 42(2), 53-76. DOI: https://doi.org/10.17981/econcuc.42.2.2021. Org.3

\begin{abstract}
Resumen
Durante el confinamiento por covid 19, se desarrolló un estudio para analizar el futuro de las relaciones interpersonales una vez se termine la emergencia sanitaria; con metodología Delphi entre directivos de mercadeo y de recursos humanos de empresas y organizaciones sin ánimo de lucro de Colombia y España. Se ejecutó en tres etapas con preguntas abiertas; se aplicó una encuesta para cerrar cada tema. La metodología Delphi permitió tanto estudiar conceptos como buscar pronósticos, que son sus fines principales; se realizó cuando se llevaba un mes de confinamiento lo que ayudó a discutir los cambios que ya se estaban viviendo y lo que los participantes creían que iba a suceder en un futuro: qué podría quedar como aprendizaje permanente para las empresas y sus grupos de interés. Los participantes consideran que la sociedad cambiará después del confinamiento; se procurará tener un equilibrio entre rentabilidad y seguridad. Los trabajadores desarrollarán creatividad, capacidad de adaptación y compromiso. La situación a mediano plazo fomentará relaciones más duraderas y fuertes con los proveedores y clientes. No se perciben grandes diferencias entre los resultados de ambos países.

Palabras clave: Covid 19; cambio; crisis; relaciones interpersonales; responsabilidad social
\end{abstract}

\begin{abstract}
A study was developed during COVID 19 confinement to foresee the future of interpersonal relationships once health emergency ended. Delphi method was used by consulting marketing and human resources managers, in private companies and nonprofit organizations from Colombia and Spain. The study was accomplished in three stages using open questions. A final survey was applied for closing every stage. The Delphi methodology allowed both studying concepts and looking for forecasts, which are its main purposes. It was carried out after a month of confinement, which allowed discussing the changes that were already being experienced and what the participants believed would happen in the future: what could remain as permanent learning for companies and their groups of interest. Participants considered that society will change after the confinement: a balance will be found between profitability and security; workers will increase creativity, adaptability and commitment. In the medium term, this situation will encourage stronger and more lasting relationships with suppliers and customers. No relevant differences were found between results from each country.

Keywords: Covid 19; change; crisis; relationships; social responsibility
\end{abstract}




\section{INTRODUCCIÓN}

Los seres humanos somos sociales por naturaleza (concepto atribuido a Aristóteles); el confinamiento realizado casi a nivel mundial por la crisis sanitaria generada por covid 19 (una enfermedad que genera problemas respiratorios serios y con alta tasa de contagio) hizo que se presentara aislamiento. En esa coyuntura de transformaciones e incertidumbre, se quiso conocer en el mismo momento del confinamiento, qué esperaban del futuro directivos de organizaciones con y sin ánimo de lucro, en cuanto a cambios en las relaciones interpersonales.

Infocaribe de Colombia e Invenies de España consultaron a expertos con el método cualitativo Delphi (por medio de la plataforma Messagenes). Los participantes plasmaban por escrito sus opiniones a preguntas abiertas a manera de debate; lo hacían de manera asincrónica. Al final de cada tema se realizó una encuesta de preguntas cerradas. El estudio se realizó entre el 16 de abril y el 26 de mayo de 2020. Participaron directores de mercadeo y de recursos humanos de cuatro segmentos: agremiaciones, organizaciones sin ánimo de lucro, grandes empresas y pymes (pequeñas y medianas empresas).

Tanto en España como en Colombia se estaba en confinamiento obligatorio durante todo el estudio, aunque en distintas fechas se fueron liberando algunos sectores de la economía para trabajar fuera de casa y se presentaron diferencias por ciudad; por ejemplo, en Barranquilla (Colombia), donde estaban varios participantes, se dieron más restricciones a la movilidad que en Bogotá, de donde también había expertos en el estudio. Para el 11 de mayo en España habían autorizado reuniones hasta de 10 personas y en Colombia hasta terminar el estudio aún no estaban autorizadas.

El primer caso de covid 19 se detectó en España el 31 de enero y en Colombia, el 6 de marzo. En Colombia se inició confinamiento nacional el 25 de marzo con 333 casos de infectados por covid 19; a 4 de mayo ya eran 7973 casos y a 24 de mayo, 21175 . En España, inició el estado de alarma el 15 de marzo, el 17 de marzo tenía 11178 casos, el 15 de abril se registraron 177633 infectados y el 24 de mayo ya eran 235772 . Por tanto, en España se dieron muchos más casos, al menos durante esta etapa. La población de los dos países es similar, alrededor de 48 millones de personas. La edad promedio de la población en España es más alta: 44 años; en Colombia es de 31 años. Cabe anotar que uno de los factores asociados a la mayor tasa de mortalidad por el virus es la vejez. Por otro lado, el PIB per cápita en Colombia es de cerca de US\$6000 y el de España, US\$30000.

En el estudio se trataron tres temas detonadores: relaciones personales dentro de la organización, relaciones con clientes y proveedores e impacto social; los participantes abrían discusiones a partir de ellos. El presente artículo presenta los resultados tanto de los debates cualitativos como de las respuestas con el instrumento estructurado. Hay una primera sección con constructos teóricos de los temas del estudio, junto con otras investigaciones relacionadas. Luego se da a conocer la definición de la metodología Delphi y las características específicas de cómo se desarrolló para el presente estudio. Después se presentan los resultados de acuerdo con los tres temas básicos del estudio. La discusión de resultados compara hallazgos con algunos de los conceptos y finalmente se llega a unas conclusiones 


\section{FundAMENTACIÓN}

\section{Relaciones interpersonales y con clientes}

Las relaciones interpersonales se definen como una conducta abierta y consciente con otro ser humano (Leary, 1957; Cornejo y Tapia, 2011). "Configuran la base de toda comunicación y se encuentran asociadas al conjunto de aptitudes que posibilitan a las personas integrarse a un grupo y ocupar en éste un lugar específico" (Vuotto y Arzadun, 2007, p. 9).

Así mismo, Valenzo-Jiménez, Martínez-Arroyo y Ávila-Carreón (2015) narran que en la década de 1920 fue cuando surgieron temas asociados al mercadeo relacional, cuyo propósito era generar relaciones rentables con los clientes. Además explican que se hizo más complicado tener buenas relaciones en la medida que las empresas crecieron y se hizo necesario tener sistemas como el Customer Relationship Management (CRM), que está diseñado para crear valor en el mantenimiento de las relaciones con los clientes, en el cual se involucran ventas, mercadeo, servicio al cliente, soporte técnico y contabilidad; se usa para influir en la percepción de los clientes hacia los productos y la organización (Plakoyiannaki \& Saren, 2006; Valenzo-Jiménez et al., 2015).

De otro lado, Barney (1991), Zahay y Griffin (2004) y Cabanelas, Cabanelas y Lorenzo (2007) anotan que en la medida que las relaciones con los clientes "sean específicas, heterogéneas y difíciles de imitar, generarán activos diferenciales de carácter intangible, que pueden sostener una ventaja competitiva” (p. 134). Igualmente, Cabanelas et al. (2007) afirman:

La generación de valor en la relación con el cliente puede derivar de la (1) obtención y aplicación de conocimiento que incremente el valor a través de la relación, la (2) habilidad para construir y mantener relaciones con los mejores clientes, la (3) organización de las relaciones con el exterior, la (4) continuidad de éstas (lo que ayuda a generar conocimiento futuro, así como confianza y adaptación mutuas) y la (5) adaptación (a los deseos y necesidades). La gestión de las relaciones con los clientes debiera centrarse en mantener y desarrollar intercambios de valor a largo plazo que deriven en un compromiso de ambas partes en la relación (pp. 134-135).

De acuerdo con Gil-Lafuente y Luis-Bassa (2011), el CRM implica estar en escucha activa de lo que los clientes opinan, reclaman y piensan, lo cual debe ser documentado, analizado y asimilado para dar respuesta y aprender. Según Bell, Auh y Smalley (2005) entendiendo cómo los clientes dan prioridad a los aspectos funcionales y técnicos del servicio puede ayudar a las empresas gestionar mejor las relaciones con sus clientes.

Entre los estudios sobre relaciones interpersonales se encuentra el señalado por Yañez, Arenas y Ripoll (2010) y realizado por Edwards y Cable (2009), "quienes encontraron que la confianza en las relaciones interpersonales tiene una influencia positiva y significativa en la satisfacción laboral" (p. 194). Yañez et al. (2010) mencionan varios estudios en que se encontró correlación entre las relaciones con pares y la satisfacción laboral, tal es el caso de Humphrey, Nahrgang y Morgeson (2007), y Chiaburu y 
Harrison (2008). Estos últimos anotan, según Yañez-Gallardo et al. (2010), que las "relaciones, caracterizadas por interacciones frecuentes, son un importante depósito de recursos emocionales y conductuales, e incluso serían más fácilmente desarrolladas que la relación con jefatura" (p. 195).

En otro sentido, desde que se declaró la pandemia, la prensa ha descrito la situación y también ha buscado predecir sus efectos. El 3 de mayo de 2020, la BBC publicó un artículo con opiniones de doce expertos, quienes predijeron mayor uso del teletrabajo, plataformas de video y automatización; también consideraron que los empleados exigirían más flexibilidad de parte de sus empleadores (BBC, 2020).

\section{Cambio}

García y Forero (2018), Malott (2001), y Armenakis y Bedeian (1999) definen el cambio organizacional como una alteración del entorno de trabajo y está relacionado con la capacidad de adaptación según motivaciones internas y externas. El contrato psicológico reconoce un intercambio transaccional como intercambio monetario, pero también como un intercambio relacional, que es un acuerdo entre ambas partes para el mantenimiento de la relación, además, incluyen aspectos motivacionales, no monetarios (Coyle-Shapiro \& Kessler, 2000; García y Forero, 2018).

Al respecto, en el estudio realizado por García \& Forero (2018) con 100 empresas colombianas en procesos de cambio, encontraron que:

Los contratos psicológicos de tipo relacional generan un impacto positivo en el clima organizacional, pero pueden estar sujetos a la percepción de ruptura por parte del empleado en la medida en que éste pueda apreciar en algún momento que el empleador no cumpla con cualquiera de sus promesas (p. 160).

\section{Responsabilidad social}

El Consejo Empresarial Mundial para el Desarrollo Sostenible (2001, citado por Díaz y Fernández, 2015) define Responsabilidad Social Corporativa como:

El compromiso continuo por parte de las empresas a comportarse de forma ética y contribuir al desarrollo económico sostenible, al tiempo que se mejora la calidad de vida de los trabajadores y sus familias, así como de la comunidad local y la sociedad en general (p. 91).

De otra parte, Gutiérrez y Navallas (2011) explican que en años recientes se considera que no es suficiente prestar atención a obtener ganancias, sino que las organizaciones deben desarrollar e informar sobre la responsabilidad social corporativa. Cuentan que la Asociación Española de Contabilidad y Administración de Empresas (Lizcano y Moneva, 2014), define que la responsabilidad social corporativa representa la contribución de la organización a la sociedad, implica un comportamiento coherente, considera fundamental el desarrollo de las personas que la conforman y la preservación del medio ambiente, tiene carácter voluntario e impacta en lo social, medioambiental y económico. 
Por otro lado, Kirschner (2006) analiza que la función social de la empresa se puede estudiar desde dos puntos de vista: relación empresa-sociedad y empresaempleados, es decir el esfuerzo que hace para "asegurar la cohesión y el dinamismo de sus empleados" (p. 139). De hecho, comenta que se suele estudiar más el medio ambiente y las relaciones con las comunidades cercanas pero que la responsabilidad social interna es muy importante; esto puede deberse a que resulta menos visible. Dentro de este aspecto, hay temas como el respeto de los jefes a los subordinados y la cooperación entre colegas.

Acerca de responsabilidad social, Salas-Arbeláez, García-Solarte y Azuero-Rodríguez (2020) realizaron un estudio en tres ciudades de Colombia con 492 pymes y hallaron que las empresas en primer lugar intentan obtener la máxima utilidad económica; en segundo lugar, manifiestan preocupación por los aspectos éticos y legales relacionados con sus grupos de interés; por ejemplo, buscan ser honestos con sus clientes y proteger el medio ambiente. La dimensión menos reportada es la de hacer actividades filantrópicas. Además, Sepúlveda et al. (2015), encontraron que la mayoría de empresas medianas y pequeñas colombianas no cuentan con actividades y políticas formales de RSE; en muchos casos porque dan prioridad a los gastos propios del negocio.

Gutiérrez y Navallas (2011) realizaron un estudio en España y encontraron que el $30 \%$ informa sobre sus programas sociales. "Los recursos humanos, la formación, la seguridad e higiene, las relaciones con clientes, las innovaciones, etcétera, son intangibles sobre los que las empresas desean informar para realzar su valor en los mercados" (p. 21). Sin embargo, hay reticencia a informar sobre el valor gastado en los programas.

Un estudio exploratorio muy reciente, presentado por Santos, Tornel y García (2020) y desarrollado también a raíz de la pandemia generada por covid 19, señala que "las prácticas de RSC producen un aumento significativo de aspectos tales como la imagen de la empresa, su reputación, la lealtad y el compromiso de los trabajadores y la satisfacción de los consumidores" (p. 9). Los autores consideran que tener prácticas de responsabilidad social puede ayudar a salir de la crisis provocada por la pandemia del covid 19; cabe anotar que el estudio se realizó a partir de recuento bibliográfico.

\section{Metodología}

Como se indicó, el método usado fue el Delphi, el cual es una metodología cualitativa que suele hacerse con grupos pequeños de no más de 30 personas divididos en subgrupos. Linston y Turoff (2002) indican que el método Delphi es un proceso de comunicación grupal. Igualmente, Somerville (2008) lo define como un proceso con varias rondas de preguntas en la que se hace una exploración abierta de un tema; uno de los objetivos es lograr consensos.

En palabras de Landeta (1999), se trata de la "obtención de una opinión grupal fidedigna a partir de un conjunto de expertos" (p. 89), que tiene una finalidad predictiva. Se atribuye su creación a Dalkney y Helmer (1963) que lo visualizaron 
como un método con cuestionarios en los que se profundiza y hay devolución de resultados a los participantes. Resulta útil para reunir personas de diferentes latitudes y permite ahorrar. Finalmente, Gordon (1994) anota que se aplica en tres situaciones:

1. Posibles previsiones sobre la ocurrencia o no de un evento y sobre cuándo se espera que ocurra.

2. Recomendaciones acerca de la conveniencia o no de que algo ocurra (¿Se debe llevar a cabo un determinado cambio? ¿Es deseable implementar un programa?).

3. Las probabilidades de que se logren las metas previstas en una situación dada, "a través de la ejecución de un programa o de la asunción de ciertas políticas" (Reguant-Álvarez \& Torrado-Fonseca, 2016, p. 90).

Además, Okoli y Pawlowski (2004) muestran ejemplos de estudios realizados con método Delphi. Entre los de hacer pronósticos está el de Kendall elaborado en 1992, que buscó predecir el rol del análisis de sistemas en el siglo XXI. Entre los estudios de concepto está el de Nambisan (1999), que desarrolló una taxonomía de acciones de diseño organizacional y mecanismos para incrementar la propensión de los usuarios de tecnología a innovar, que fue realizado entre directivos de diversas industrias.

En Colombia, Rodríguez y Gutiérrez (2019) utilizaron el método Delphi para hacer un análisis prospectivo de la tecnología en el trabajo hacia 2050; participaron directivos universitarios, investigadores y asesores. Hallaron que la sociedad debe estar consciente que los cambios afectarán la vida cotidiana y que la transformación digital generará oportunidades; lo cual traerá cambios culturales.

En este caso específico, se hizo en tres rondas con preguntas abiertas, a partir de las cuales surgieron más preguntas abiertas de parte de los expertos o de los moderadores. Los participantes plasmaban por escrito sus opiniones a preguntas abiertas a manera de debate; lo hacían de manera asincrónica. El estudio se realizó entre el 16 de abril y el 26 de mayo de 2020; el primer tema se trató hasta el 28 de abril; el segundo hasta el 8 de mayo y después se desarrolló el tercero. Fueron cuatro grupos; cada uno tuvo un moderador, quien no opinaba para evitar sesgo. Hubo moderadores tanto de España como de Colombia; los moderadores podían ingresar a las salas de sus compañeros con el fin de observar la dinámica de los otros grupos $y$, eventualmente, para aclarar algún vocablo de uso regional.

Además, una persona estuvo a cargo de visitar todas las salas para hacer seguimiento al proceso. Las preguntas iniciales de cada tema eran iguales para todos los grupos y luego se ajustaban según la dinámica de cada grupo. Al terminar cada tema se hizo un cuestionario estructurado igual para todos los grupos. Se hicieron devoluciones parciales; en algunos momentos se informaron de temas tratados por otros grupos, se procuró que estas devoluciones fueran temas detonantes, pero no con detalles para dejar en libertad de opinar. Durante el proceso los expertos opinaron sobre comentarios de sus compañeros. Al final se entregaron a los expertos conclusiones reuniendo resultados de todos los grupos. 
Dados los temas, se definió el perfil como personas involucradas con recursos humanos, área comercial o mercadeo o con ambos temas, en nivel gerencial (de la sede principal o regional). Se buscó que las personas tuvieran varios años de experiencia.

Participaron directores de mercadeo, de recursos humanos o gerentes generales de cuatro segmentos: agremiaciones, organizaciones sin ánimo de lucro, grandes empresas y pymes (pequeñas y medianas empresas). Se contó con participantes de diversos sectores económicos: funerario, construcción, textil, tecnología de la información, climatización, transporte, banca, producción de alimentos, industria papelera, producción de acero, comercialización de muebles, ferretería, publicidad, logística y cosmética. En cuanto a organizaciones sin ánimo de lucro, entidades que trabajan en los siguientes frentes: actividad cultural, apoyo psicológico, desarrollo comunitario y medio ambiente. El segmento de agremiaciones tuvo miembros de cámaras de comercio, asociaciones de directivos y asociaciones empresariales.

El grupo de empresas grandes contó con siete expertos; el de empresas pequeñas y medianas tuvo ocho; el de organizaciones sin ánimo de lucro, cinco y el de agremiaciones, cuatro. Para un total de 24 participantes, de los cuales diez estaban en España y catorce en Colombia. Algunos otros ingresaron a la plataforma, pero no se involucraron.

No todos los participantes que dieron sus opiniones con las preguntas abiertas contestaron la encuesta. La encuesta 1 tuvo como muestra 14 válidas de los cuatro grupos; de hecho, por tener pocas encuestas de cada segmento, el análisis se hizo conjunto. El cuestionario tenía 14 preguntas cerradas con escala de cinco puntos (seguro que no pasará, probablemente no pasará, seguirá igual, probablemente pasará y seguro que pasará); de autoaplicación, vía plataforma Messagenes (la misma en que se hacía el debate abierto).

La encuesta 2 contó con 11 casos válidos de los cuatro grupos; en este caso se hicieron algunos análisis por país, por tener diferencias significativas aunque se trata de una muestra pequeña. El cuestionario tuvo 20 preguntas cerradas, que surgieron de las propias opiniones de los participantes y que también tenía escala de cinco puntos. La encuesta que cerró el tercer tema, tuvo como muestra 14 unidades de los cuatro segmentos. Este es el único cuestionario que tuvo una pregunta abierta, que se convirtió en múltiple pues cada persona dio hasta seis respuestas, que fueron codificadas. Además de la pregunta abierta, se hicieron cinco cerradas; fueron menos preguntas pues el último tema tuvo menor acogida en el debate abierto. Para el análisis cuantitativo se usó SPSS versión 26 y el gráfico fue hecho en Excel 2013.

\section{AnÁlisis DE Resultados}

\section{Primer tema: Relaciones dentro de la organización}

Participantes tanto del grupo de empresas grandes como de pymes señalan que las personas cambian de manera constante y, por tanto, pasa igual en las relaciones interpersonales. Lo que sucede es que la crisis por covid 19 fue una 
transformación abrupta que ha dificultado la adaptación. Uno de los grandes problemas es que se espera que las personas estén distanciadas en el contacto físico y eso es contrario a las costumbres de ambos países. En estos grupos y el de organizaciones sin ánimo de lucro hay preocupaciones como que los dueños de las empresas quieran reemplazar empleados por robots $u$ otros elementos tecnológicos que permitan manejar mejor la crisis sanitaria; aunque reconocen que gracias a la tecnología es que se ha podido sacar adelante a la organización en medio de la coyuntura.

En el grupo de organizaciones sin ánimo de lucro, un participante español comenta que "toda la sociedad deberá convivir en un complicado equilibrio entre rentabilidad, riesgo y seguridad; lo que viene siendo la acreditación de un puesto de trabajo seguro. La dinámica de la sociedad cambiará”. Varios participantes manifestaron su acuerdo con esta posición.

También se comenta en este grupo que los cambios en este tipo de organizaciones deben ir acompañados de modificaciones en la legislación, es decir en las relaciones con los gobiernos pues su trabajo suele estar muy atado a estos; en el caso de España, explican, por ejemplo, que las condiciones de los pliegos de licitación dificultan contratar asesorías externas. A futuro, consideran que las entidades no lucrativas deben hacer ver a la sociedad en general que su labor es útil y dirigida a necesidades básicas y a las administraciones locales, que las fundaciones son fuertes en áreas que a los gobiernos les cuesta suplir.

Otros cambios esperados por miembros de los diferentes segmentos son:

En cuanto a perfil laboral:

a. Hay habilidades personales que serán más importantes como la flexibilidad, el trabajo en equipo, la creatividad, la agilidad para tomar decisiones, la capacidad de adaptación y el compromiso.

b. Posiblemente las empresas tenderán a contratar personas con menor perfil de riesgo de complicarse con covid 19 como los jóvenes que sean activos físicamente.

En cuanto a relaciones empresas-trabajadores:

a. Trabajadores y empresas velarán por sus intereses y se pueden presentar fricciones.

b. Se deberán establecer nuevos acuerdos entre empresa y trabajador ante los cambios constantes.

c. Las empresas deberán confiar más en sus empleados, por ejemplo para trabajar fuera de oficina.

d. Cambiarán las estrategias organizacionales y el clima laboral; se trabajará bajo presión emocional.

e. Las empresas deben romper paradigmas e innovar.

f. Las empresas valorarán más al recurso humano y las personas valorarán lo que tienen. 
En cuanto a relaciones con colegas:

a. La situación de crisis ha provocado mayor preocupación por el compañero de trabajo como ser humano.

b. Las personas extrañan la interacción social y apenas haya cura para la enfermedad o mejore la situación sanitaria, querrán retomarlas; que se den relaciones de amistad y compañerismo.

Un participante de entidades sin ánimo de lucro afirma que "si algo va a cambiar esta pandemia es la de demostrar que un trabajador comprometido rinde probablemente más si puede combinar el teletrabajo con sesiones presenciales de calidad. Así, ganaremos todos". Otra persona de este grupo comenta que el teletrabajo implica cambios en la actitud, los paradigmas y los hábitos; explica que entre sus colaboradores hay una población de edad avanzada que no tenía experiencia con el uso de equipos de cómputo, lo que ha implicado desarrollar herramientas y contenidos pedagógicos en un corto tiempo; además trabajan en lugares de pocos recursos económicos y aislados, que cuentan con poca conectividad.

Otra participante de fundaciones hace ver que el teletrabajo, al menos en Colombia, puede prestarse para que se disfrace trabajo como independiente o autónomo cuando en realidad se trata de un contrato laboral en que las empresas deben asumir las prestaciones sociales (servicio de salud y ahorro para pensión). Y alguien más del segmento aclara que hay personas que por su personalidad y relaciones familiares no les es posible adaptarse al teletrabajo a largo plazo; caso de los muy dependientes de las relaciones sociales y de los que si pasan mucho tiempo con su familia "no se la aguantan".

De hecho, en este grupo se dio un debate porque algunos consideran que se puede ser más productivo en casa al tener mejor calidad de vida (como un hombre que lleva nueve años con teletrabajo) y otros estiman que no se alcanza la productividad sin el contacto con los colegas (es más la posición de quienes tomaron el teletrabajo de repente y sin mayor planeación).

Algunas de las predicciones en cuanto a los clientes son: mayor inversión en la búsqueda de clientes nuevos; búsqueda de maneras diferentes de vender y de formas de pago distintas al efectivo. Se requerirá convencer a los clientes de invertir siendo que seguramente bajaron sus recursos económicos; esto aplica tanto para cliente corporativo como usuario final.

En cuanto a la encuesta que cerró este primer tema, en la Tabla 1 se presentan las preguntas en que hubo más acuerdo entre los expertos, pues el error estándar de la media fue inferior; el valor más bajo es para 'se incrementarán las políticas de seguridad y prevención de riesgos laborales', 'será necesario incrementar la ciberseguridad', 'será necesario mejorar la formación de las personas que teletrabajen' y 'será necesario mejorar los procesos de comunicación interna', que es de 0.097; las dos primeras cuestiones asociadas al tema de seguridad y las otras a temas de recursos humanos. En las cuatro preguntas, la media (escala de 1 a 5 , donde 5 es 'seguro que pasará') fue de 4.86 , que es la más alta registrada y que demuestra que los exper- 
tos consideran que son hechos que seguramente se darán después de terminada la etapa principal de la emergencia sanitaria.

En estas preguntas la calificación mínima fue 4 y la máxima 5; la moda y la mediana fueron 5. Igualmente, entre el cuartil 1 (o percentil 25) y el cuartil 3 (percentil 75) la mitad central es 5 . Así mismo, una desviación estándar de menos de 0.85 da cuenta de que en estos temas hubo consenso entre los expertos.

TABLA 1.

Estadísticos de respuestas en que hubo mayor acuerdo entre los expertos en encuesta 1.

\begin{tabular}{|c|c|c|c|c|c|}
\hline \multicolumn{6}{|c|}{ Estadísticos } \\
\hline & & $\begin{array}{c}\text { Se incrementarán las } \\
\text { políticas de seguridad } \\
\text { y prevención de } \\
\text { riesgos laborales }\end{array}$ & $\begin{array}{c}\text { Será necesario } \\
\text { mejorar la formación } \\
\text { de las personas que } \\
\text { teletrabajen }\end{array}$ & $\begin{array}{l}\text { Será necesario } \\
\text { incrementar la } \\
\text { ciberseguridad }\end{array}$ & $\begin{array}{c}\text { Será necesario } \\
\text { mejorar los procesos } \\
\text { de comunicación } \\
\text { interna }\end{array}$ \\
\hline $\mathrm{N}$ & Válido & 14 & 14 & 14 & 14 \\
\hline \multicolumn{2}{|l|}{ Media } & 4.86 & 4,86 & 4.86 & 4,86 \\
\hline \multicolumn{2}{|c|}{$\begin{array}{l}\text { Error estándar de la } \\
\text { media }\end{array}$} & 0.097 & 0.097 & 0.097 & 0.097 \\
\hline \multicolumn{2}{|l|}{ Mediana } & 5.00 & 5,00 & 5.00 & 5.00 \\
\hline \multicolumn{2}{|l|}{ Moda } & 5 & 5 & 5 & 5 \\
\hline \multicolumn{2}{|c|}{ Desv. Desviación } & 0.363 & 0.363 & 0.363 & 0.363 \\
\hline \multicolumn{2}{|l|}{ Varianza } & 0.132 & 0.132 & 0.132 & 0.132 \\
\hline \multicolumn{2}{|l|}{ Mínimo } & 4 & 4 & 4 & 4 \\
\hline \multicolumn{2}{|l|}{ Máximo } & 5 & 5 & 5 & 5 \\
\hline \multirow{3}{*}{ Percentiles } & 25 & 5.00 & 5.00 & 5.00 & 5.00 \\
\hline & 50 & 5.00 & 5.00 & 5.00 & 5.00 \\
\hline & 75 & 5.00 & 5.00 & 5.00 & 5.00 \\
\hline
\end{tabular}

Fuente: Elaboración propia.

Teniendo en cuenta todos los promedios, los aspectos que los expertos más predicen que se llevarán a cabo en la etapa post covid 19 son:

a. Se incrementarán las políticas de seguridad y prevención de riesgos laborales.

b. Será necesario mejorar la formación de las personas que teletrabajen.

c. Será necesario incrementar la ciberseguridad.

d. Será necesario mejorar los procesos de comunicación interna.

e. Será necesario modificar los procesos de trabajo.

f. Será necesario entender las consecuencias del teletrabajo para el personal.

Así es que los temas de seguridad y teletrabajo predominan pero también saben que los cambios implicarán ajustar procesos y saber comunicarlos. 


\section{Segundo tema: Relaciones con los clientes y proveedores}

En la parte de preguntas abiertas, el tema de las relaciones con clientes potenciales, clientes actuales, proveedores y usuarios fue muy relacionado por los participantes con lo ya anotado para las relaciones dentro de la empresa, pues sienten que se afectan de la misma forma con el confinamiento y las medidas de distanciamiento social que van a venir una vez termine la cuarentena. De hecho, el asunto de los clientes ya había surgido en el primer tema con lo siguiente. Personas de pymes y empresas grandes expresaron que se debe buscar maneras de fortalecer las relaciones con los clientes a pesar de no poderlo hacer personalmente pues cambiarán las relaciones humanas en general y también hay que buscar canales no presenciales para atender clientes.

En el segmento de entidades sin ánimo de lucro hicieron notar que su trabajo en muchas ocasiones implica el abrazo, además de la confidencia y las emociones, como herramienta fundamental de su labor, por lo cual a futuro esperan que muchas actividades no se hagan a distancia; por supuesto, en este momento de confinamiento obligatorio, el video ha sido mejor que nada para llegar a sus usuarios.

También en este grupo explican que, por la pandemia, todos los seres humanos están en un mismo nivel; hay conciencia de que las acciones de uno afectan a todos, se siente sensibilidad y vulnerabilidad. Eso hace que la relación con proveedores sea más fraternal, así como sucede con trabajadores, familiares y vecinos. En esta situación contribuyen las videoconferencias pues los niños u otros miembros de la familia entran a participar y proveedores y clientes terminan conociéndolos e interesándose en su bienestar. Las comunicaciones se tornan transparentes y se pueden mostrar los malos momentos por los que estamos pasando; contrario a lo que sucede en otras épocas en que la idea es manifestarse fuertes para así obtener mejor posicionamiento en la negociación o mejor imagen ante los nuevos clientes.

Así, ha sido más fácil pedir apoyo a los proveedores cuando no se puede pagar en los tiempos acostumbrados u otras dificultades. En todo caso, la situación a mediano plazo fomentará tener relaciones más duraderas y fuertes con los proveedores y clientes. La prioridad es buscar maneras de poder operar, no continuar paralizados y en eso coinciden con los clientes y proveedores; ayudarse mutuamente como buscar hacer canjes o recibir donaciones de bienes o servicios, así no se generan ingresos pero tampoco gastos. Los proveedores se terminan convirtiendo en financiadores. Además, eso se debe comunicar a los financiadores y proveedores; "hacerles partícipes de lo que hacemos, de logros concretos; buscar retornos emocionales tangibles". Por supuesto, aclaran que algunos clientes no han sido empáticos y esto puede llevar a romper relaciones.

Cambios esperados en las relaciones con clientes, proveedores y usuarios al salir del confinamiento:

En cuanto a tecnología:

a. Hacer un mayor uso de la tecnología, revisando los contenidos de las página Web, con automatización de procesos y atención virtual, acompañada de alineamiento cultural del recurso humano. 
En cuanto a comunicación:

a. Todo lo que se pueda se hará vía telefónica o virtual pero hay casos en que la venta debe hacerse personal como la construcción, en la que hay que tomar medidas y evaluar el terreno.

b. Se incrementará el uso de catálogo y pedido vía Internet.

c. Los catálogos pueden apoyarse de videos explicativos de cómo funcionan los productos y de fotografías.

d. Mayor atención a las redes sociales.

e. Debe ser fácil localizar a las empresas en el entorno virtual.

f. Mostrar el lado humano de la empresa: solidaridad hacia el cliente e informarle que la empresa lo necesita.

g. Consistencia entre los diferentes canales; omnicanal.

h. Gestión de trámites de manera virtual.

Aspectos que no cambiarán en relaciones con clientes y proveedores post covid 19:

a. Confianza sigue siendo muy importante.

b. Se parte de que la calidad es lo primero.

c. Relaciones con proveedores del área de investigación y desarrollo, diseño y fabricación tenderán a seguir siendo personales porque implica tocar materiales y productos.

De otro lado, en el cuestionario cerrado aplicado al final de este tema, se puede observar lo siguiente. En la Tabla 2 se presentan las preguntas de la segunda encuesta en que hubo más acuerdo entre los expertos, pues el error estándar de la media fue inferior. Ellas son:

a. Se incrementarán las relaciones vía teléfono e Internet (0.091).

b. Será necesario implementar la figura de asesor de venta virtual para los clientes que no se manejan con las nuevas tecnologías (0.091).

c. Se reducirán los eventos presenciales (0.091).

d. Se incrementará la importancia del área de comunicación (0.091).

e. Será necesario formar al equipo de ventas en entornos virtuales (0.122).

f. Más e-commerce (0.152).

g. Se deberá invertir en departamentos de atención virtual al cliente (0.152).

Se puede observar que en todas estas preguntas la escala osciló entre 4 y 5 y la moda es 4 o 5 . Las dos medias más altas son las referentes a las relaciones vía Internet y teléfono y que se incrementará la importancia del área de comunicación (con 4.91). También es muy alta en 'formar equipos de ventas en entornos virtuales' (4.82). En dos preguntas la media es cercana a 4, o sea que no los consideran tan vitales como otros pero hay mucha coincidencia en las opiniones, dada la poca 
dispersión; ellas son: 'implementar asesor de venta virtual para clientes que no manejan nuevas tecnologías' y 'se reducirán eventos presenciales' (4.09). En tres casos el cuartil 1 y el 3 son 5, lo que muestra la uniformidad hacia la nota más alta o de mayor importancia.

TABLA 2.

Estadísticos de respuestas en que hubo mayor acuerdo entre los expertos en encuesta 2.

\begin{tabular}{|c|c|c|c|c|c|c|c|c|}
\hline \multicolumn{9}{|c|}{ Estadísticos } \\
\hline & & $\begin{array}{c}\text { Se } \\
\text { incrementarán } \\
\text { las relaciones } \\
\text { vía teléfono e } \\
\text { internet }\end{array}$ & $\begin{array}{c}\text { Más } \\
\text { e-com_- } \\
\text { merce }\end{array}$ & $\begin{array}{c}\text { Asesor } \\
\text { de venta } \\
\text { virtual } \\
\text { para } \\
\text { clientes que } \\
\text { no manejan } \\
\text { nuevas } \\
\text { tecnologías }\end{array}$ & $\begin{array}{c}\text { Se } \\
\text { reducirán } \\
\text { los eventos } \\
\text { presenciales }\end{array}$ & $\begin{array}{c}\text { Se } \\
\text { incrementará } \\
\text { la } \\
\text { importancia } \\
\text { del área de } \\
\text { comunicación }\end{array}$ & $\begin{array}{c}\text { Formar } \\
\text { al equipo } \\
\text { de } \\
\text { ventas } \\
\text { en } \\
\text { entornos } \\
\text { virtuales }\end{array}$ & $\begin{array}{l}\text { Invertir en } \\
\text { departamentos } \\
\text { de atención } \\
\text { virtual al } \\
\text { cliente }\end{array}$ \\
\hline $\mathrm{N}$ & Válido & 11 & 11 & 11 & 11 & 11 & 11 & 11 \\
\hline Media & & 4.91 & 4,64 & 4.09 & 4.09 & 4.91 & 4.82 & 4.64 \\
\hline $\begin{array}{l}\text { Error están } \\
\text { media }\end{array}$ & ar de la & 0.091 & 0.152 & 0.091 & 0.091 & 0.091 & 0.122 & 0.152 \\
\hline Mediana & & 5.00 & 5.00 & 4.00 & 4.00 & 5.00 & 5.00 & 5.00 \\
\hline Moda & & 5 & 5 & 4 & 4 & 5 & 5 & 5 \\
\hline Desv. Desvi & ción & 0.302 & 0.505 & 0.302 & 0.302 & 0.302 & 0.405 & 0.505 \\
\hline Varianza & & 0.091 & 0.255 & 0.091 & 0.091 & 0.091 & 0.164 & 0.255 \\
\hline Mínimo & & 4 & 4 & 4 & 4 & 4 & 4 & 4 \\
\hline Máximo & & 5 & 5 & 5 & 5 & 5 & 5 & 5 \\
\hline \multirow{3}{*}{ Percentiles } & 25 & 5.00 & 4.00 & 4.00 & 4.00 & 5.00 & 5.00 & 4.00 \\
\hline & 50 & 5.00 & 5.00 & 4.00 & 4.00 & 5.00 & 5.00 & 5.00 \\
\hline & 75 & 5.00 & 5.00 & 4.00 & 4.00 & 5.00 & 5.00 & 5.00 \\
\hline
\end{tabular}

Fuente: Elaboración propia.

Teniendo en cuenta todos los promedios, los aspectos que los expertos más predicen que se llevarán a cabo en la etapa post covid 19, en cuanto a relaciones con clientes y proveedores son:

a. Se incrementarán las relaciones vía teléfono e Internet.

b. Se incrementará la importancia del área de comunicación.

c. Será necesario formar el equipo de ventas en entornos virtuales.

d. Se deberá invertir en departamento de atención virtual al cliente.

e. Más e-commerce.

f. Incremento de las ventas por redes sociales.

Varios de los expertos se dedican al área comercial, que es afín al tema. En general, las respuestas más contundentes tuvieron que ver con manejar las relaciones con clientes y proveedores en entorno virtual. 
En la Figura 1 se aprecia que el 91\% considera que 'seguro pasará' que las relaciones vía teléfono e Internet se incrementarán, al igual que la importancia de las comunicaciones. También es muy alta la proporción de esa respuesta para la 'formación del equipo de ventas en entornos virtuales' (82\%). Para 'la recepción de mercancías se ralentizará por los protocolos', el mayor porcentaje corresponde a seguirá igual (46\%). La única respuesta de 2 es para la subida de precios de materias primas y nadie contestó 1 en la escala.

\section{Relaciones con clientes y proveedores}

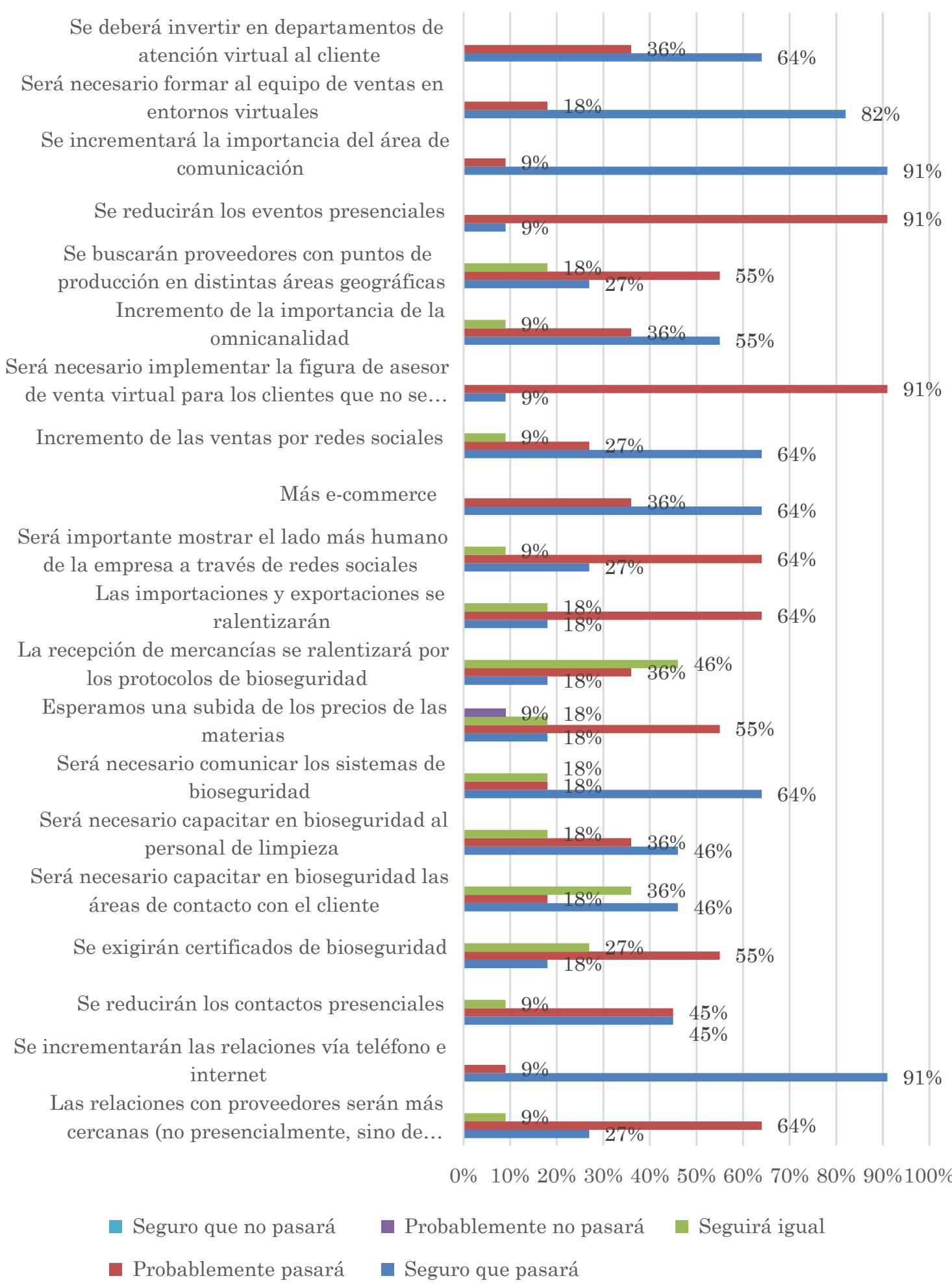

Figura 1. Respuestas relaciones con clientes y proveedores.

Fuente: Elaboración propia. 
Aunque son pocas las unidades para confiar en resultados por segmento, se observa que los expertos de España tendieron a pensar más que los certificados de bioseguridad seguirán igual (43\%); en cambio, esta respuesta no la dio nadie en Colombia, sino la equivalente a 4 o 5 . Es posible que la diferencia se dé porque en la actualidad (antes de crisis sanitaria) exigen más certificados en España que en Colombia.

Algo similar ocurre con las afirmaciones 'será necesario capacitar en bioseguridad las áreas de contacto con el cliente' y 'será necesario capacitar en bioseguridad al personal de limpieza', dado que el 57\% de los expertos españoles consideró que 'seguirá igual', el 29\% que 'probablemente pasará' y el 14\% que 'seguro pasará; esta última fue la respuesta de todos los colombianos. De hecho, la prueba de chi cuadrado muestra un valor de significancia de 0.023 , es decir que es inferior a 0.05 y denota una diferencia significativa por país.

En esto también pudo influir que el desconfinamiento se dio un poco más rápido en España que en Colombia; este tema se trató en el estudio a principios de mayo cuando en España se estaban liberando más sectores de la economía, por tanto, es posible que estuviera más avanzada en protocolos que en Colombia. Estos datos se corroboran con la prueba t para igualdad de medias entre los grupos de ambos países; para el caso del tema del personal de limpieza el intervalo de confianza al 95\% no contiene cero y por tanto hay diferencia significativa (el intervalo es de 0.344 a 1.942 al asumir varianzas iguales); situación que se repite con el tema de certificados de seguridad (0.150 a 1.707). Se anota que en la prueba de Levene la probabilidad asociada al estadístico es mayor a 0.05 , por lo que se deben asumir varianzas iguales (Tabla 3).

TABLA 3.

Prueba t por país en aspectos de la encuesta 2.

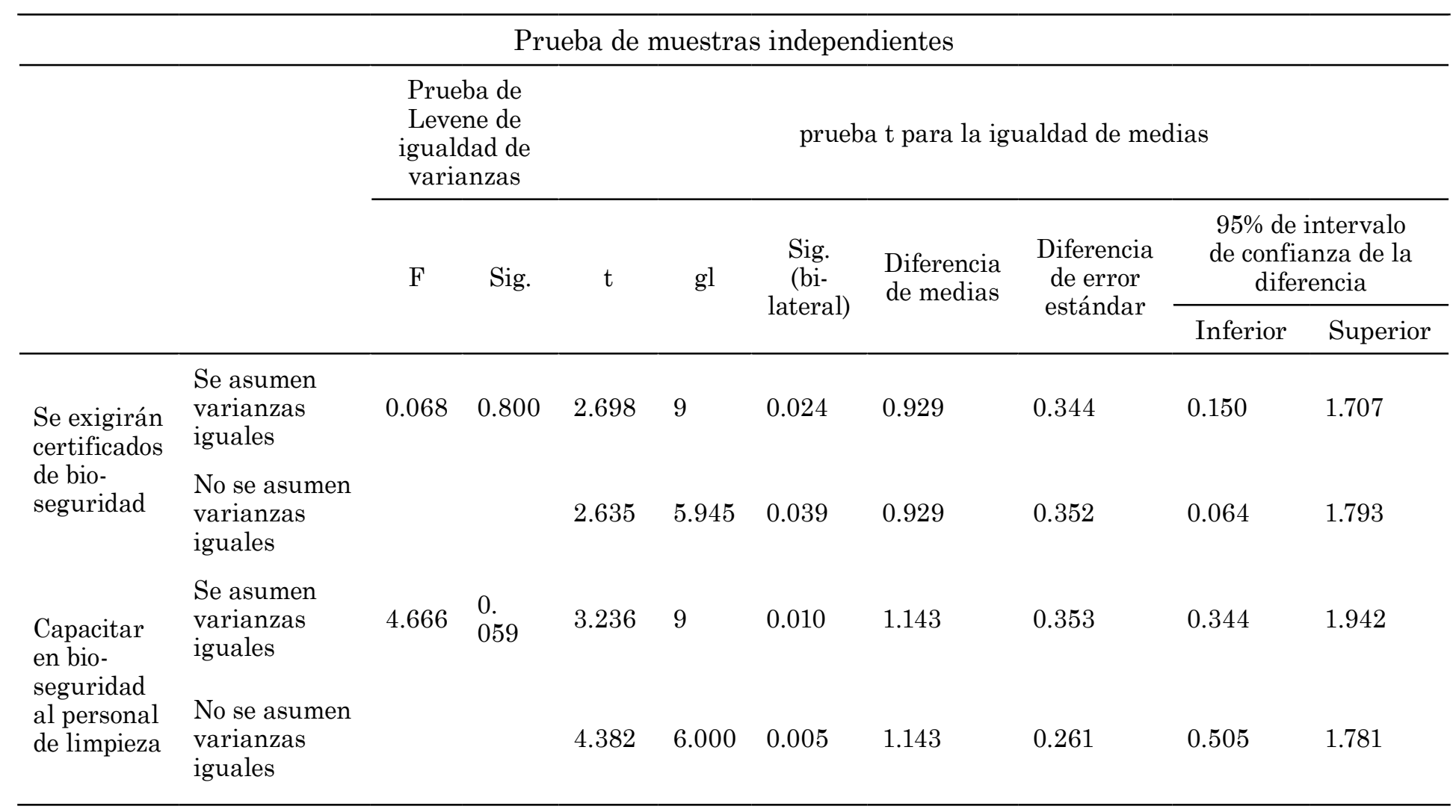

Fuente: Elaboración propia. 


\section{Tercer tema: Impacto social}

Es un tema que despierta polémica pues puede verse como apoyo a campañas solidarias, necesarias en este momento coyuntural, pero también de múltiples otras formas como que la responsabilidad social empieza por la empresa. En el tema de impacto social, los participantes de empresas grandes y pymes consideran que la primera responsabilidad es para con los empleados; la prioridad no debe ser la rentabilidad ni la compra inmediata sino buscar maneras de apoyar a la comunidad y a los clientes, protegiendo el empleo.

Con las entidades sin ánimo de lucro el tema es diferente pues su actividad de por sí tiene enfoque social. Han tratado de medir su impacto social con indicadores como valorar la efectividad de sus actividades, pero no ha sido fácil definir los criterios. Medir es importante para mostrar el retorno de la inversión a los financiadores. Otro punto para ellos es que es importante trabajar en mostrar lo que hacen, pues ser visibles es una forma de generar impacto.

Fue la única encuesta de cierre de tema con pregunta abierta, se trató de: ¿En qué nos centraremos en cuanto a impacto social? Los temas generales fueron: finanzas, empleo, salud, tecnología, mercados, proyectos sociales como tal y cuidado de medio ambiente. La respuesta más común es que las entidades se centrarán en disminuir costos (29\% de los casos); otras que se repiten son retención de empleo, flexibilidad horaria, normas de salud y transformación de la comunidad (21\%).

TABla 4.

Estadísticos encuesta 3.

\begin{tabular}{|c|c|c|c|c|c|c|}
\hline \multicolumn{7}{|c|}{ Estadísticos } \\
\hline & & $\begin{array}{c}\text { Conservar los } \\
\text { puestos de } \\
\text { trabajo }\end{array}$ & $\begin{array}{l}\text { Ampliar } \\
\text { las redes } \\
\text { sociales }\end{array}$ & $\begin{array}{l}\text { Donaciones } \\
\text { a ONG's }\end{array}$ & $\begin{array}{l}\text { Cooperación } \\
\text { con entidades } \\
\text { no lucrativas }\end{array}$ & $\begin{array}{c}\text { Apoyar el } \\
\text { emprendimiento }\end{array}$ \\
\hline $\mathrm{N}$ & Válido & 14 & 14 & 14 & 14 & 14 \\
\hline \multicolumn{2}{|l|}{ Media } & 4.36 & 4.50 & 3.43 & 3.71 & 4.14 \\
\hline \multicolumn{2}{|c|}{ Error estándar de la media } & 0.225 & 0.203 & 0.137 & 0.221 & 0.231 \\
\hline \multicolumn{2}{|l|}{ Mediana } & 4.50 & 5.00 & 3.00 & 3.50 & 4.00 \\
\hline \multicolumn{2}{|l|}{ Moda } & 5 & 5 & 3 & 3 & 4 \\
\hline \multicolumn{2}{|c|}{ Desv. Desviación } & 0.842 & 0.760 & 0.514 & 0.825 & 0.864 \\
\hline \multicolumn{2}{|l|}{ Varianza } & 0.709 & 0.577 & 0.264 & 0.681 & 0.747 \\
\hline \multicolumn{2}{|l|}{ Mínimo } & 2 & 3 & 3 & 3 & 2 \\
\hline \multicolumn{2}{|l|}{ Máximo } & 5 & 5 & 4 & 5 & 5 \\
\hline \multirow{3}{*}{ Percentiles } & 25 & 4.00 & 4.00 & 3.00 & 3.00 & 4,00 \\
\hline & 50 & 4.50 & 5.00 & 3.00 & 3.50 & 4.00 \\
\hline & 75 & 5.00 & 5.00 & 4.00 & 4.25 & 5.00 \\
\hline
\end{tabular}

Fuente: Elaboración propia. 
Este tema tuvo menos opiniones en el debate y por ello la encuesta de cierre fue más breve, se indagó sobre qué asuntos tienen mayor probabilidad de ocurrencia en la etapa pos pandemia. La menor variabilidad se dio en la frase 'donaciones a ONG' (0.137 de error estándar de la media); también para este ítem corresponde la media más baja con 3.43, es decir que es lo que los expertos ven como menos probable.

Igualmente, la media está debajo de 4 para el caso de 'cooperación con entidades no lucrativas'. En cambio, 'ampliar las redes sociales' obtuvo el mayor promedio con 4.5. También es alta la media para 'conservar los puestos de trabajo' (4.36). Además, el apoyo al emprendimiento es visto como algo posible (media de 4.14; aunque el rango de respuesta fue de 2 a 5) y allí podría estar la clave de las labores relacionadas con responsabilidad social. Esto se encuentra en la Tabla 4.

Hay una relación entre dos de los temas, pues en la prueba de chi cuadrado de Pearson el valor de significancia es 0.011: el 71\% de quienes contestaron que 'mantener los puestos de trabajo será una prioridad' dio una calificación de 4 o 5 para 'apoyar el emprendimiento'.

\section{Comparativo cuestionarios cerrados de los tres temas}

Para el primer tema la media fue de 4.6 entre todas las preguntas y encuestados; para el segundo tópico, 4.31 y para el tercero, 4.03. Entonces se consideró más factible que se den los tópicos del primer tema (relaciones dentro de la organización) y los ítems evaluados del último tema son los que tienen menos probabilidad de ocurrencia post covid 19. también, si se comparan los promedios del error estándar de la media en cada tema, se tiene que es menor para el primer tema, o sea en el que hubo más acuerdo (0.158) y el más alto fue en el tercero, donde hubo menos acuerdo, con 0.203 .

El segundo tema tuvo un promedio de 0.183 en dicha medida de dispersión. Pudo contribuir que, para el tercer tema, tal vez por cansancio, hubo menos debate y ello pudo incidir en la presencia de consenso; también que entre los participantes había dos grupos de organizaciones sin ánimo de lucro (agremiaciones y el grupo propiamente de entidades sin ánimo de lucro), así es que pueden percibir diferente el tema de las relaciones con $\mathrm{ONG}$ a como puedan acercarse las empresas, tópico asociado a impacto social, que fue el último.

\section{Discusión De Resultados}

Una de las coincidencias más fuertes del estudio se da con lo expresado por los predictores de BBC (2020); en ambos casos concuerdan con que habrá mayor uso del teletrabajo al terminar el confinamiento; también que se usarán más plataformas de video y automatización y que los empleados buscarán mayor flexibilidad de parte de sus empleadores.

Los participantes del estudio mencionaron que en el nuevo modelo de interacción hacía falta la relación física con los compañeros de trabajo pero a la vez, ha habido una preocupación por los sentimientos de los compañeros. Esto es comparable con 
los estudios de Chiaburu y Harrison (2008), y Edwards y Cable (2009), mencionados por Yañez et al. (2010), quienes muestran que los intercambios frecuentes con los compañeros son un motivo de satisfacción laboral.

En el tema de los clientes, se encontró que hay tendencia a considerar que después del confinamiento las relaciones serán más sólidas, pues de hecho en la cuarentena ha habido mayor transparencia al comunicarse con ellos; lo cual también influye en el impacto social. Esto sería congruente con lo expresado por Cabanelas et al., (2007) sobre incrementar valor por medio de la relación con los clientes; su construcción y mantenimiento. Igualmente, los participantes señalan que la confianza seguirá siendo algo vital en las relaciones con los clientes pos covid 19, que es algo también descrito por Cabanelas et al. (2007).

Otro punto es que los participantes estiman que la responsabilidad social debe partir de la relación con los empleados; en este momento de coyuntura es vital conservar los empleos. El contrato psicológico mencionado por Coyle en García y Forero (2018) tiene que ver con ello pues indica que en la relación empresa empleador hay un intercambio relacional con aspectos motivacionales más allá de los monetarios. Específicamente se puede comparar con los estudios de García \& Forero (2018) realizados en Colombia en que indican que los empleados esperan que no haya ruptura por parte del empleador y cumpla sus promesas.

También se encontró que se debe asegurar cohesión con los empleados por parte de la empresa, que coincide con lo expuesto por Kirschner (2006). Este punto del presente estudio revela más que una predicción, un deseo; de hecho, durante el confinamiento uno de los participantes perdió su empleo. Así mismo, dicha intención de asociar responsabilidad social con el compromiso de los trabajadores es coherente con la propuesta de Santos et al. (2020) acerca de estimular prácticas de responsabilidad social para mitigar la crisis generada por covid 19 .

Los miembros de entidades sin ánimo de lucro participantes indicaron que es importante dar a conocer lo que hacen, ser más visibles; incluso una persona lo llamó 'cacarear el huevo'. Sepúlveda et al. citados por Salas-Arbeláez et al. (2020), encontraron que las empresas colombianas no cuentan con actividades formales de responsabilidad social; y Gutiérrez y Navallas (2011) encontraron que en España apenas el 30\% de las empresas informa sobre sus procesos sociales, así es que esta idea llenaría este vacío por parte de los receptores de las actividades, como son las entidades sin ánimo de lucro.

Por otra parte, en la aplicación del método Delphi en el presente estudio se pudieron visualizar aspectos como: a) permitió la comunicación grupal, como indican Linston y Turoff (2002); b) se puede realizar en varias rondas (Somerville, 2008); c) facilitó establecer contacto con personas de diferentes latitudes y a un bajo costo, tal y como fue visualizado por sus creadores Dalkney y Helmer (1963); d) sirvió para medir el ambiente durante el confinamiento y además para establecer posibles previsiones, como lo han establecido Gordon (1994), Reguant-Álvarez y Torrado-Fonseca (2016) y Rodríguez y Gutiérrez 2019). Es decir, facultó tanto estudiar conceptos como buscar pronósticos, como lo explicado por los estudios de Nambisan y Kendall (citados en Okoli \& Pawlowski, 2004). 


\section{CONCLUSIONES y RECOMENDACIONES}

En los cuatro grupos de participantes consideran que la sociedad cambiará después del confinamiento; se procurará tener un equilibrio entre rentabilidad y seguridad. La seguridad está dada por elementos como barreras para el distanciamiento social o mascarillas pero también con la actitud de las empresas; que muestren compromiso con sus empleados y con la sociedad, que sean solidarias y propendan por el bienestar común antes que por sus propios intereses. Se percibe un ambiente de unión que permea a las organizaciones; hay mayor preocupación por el bienestar de los compañeros. Es algo positivo de la situación y que debe perdurar.

La crisis sanitaria ha hecho que los trabajadores deban desarrollar habilidades como la creatividad, la capacidad de adaptación y el compromiso.

Las circunstancias han llevado a que las relaciones entre empresas y trabajadores sean flexibles, por un lado por mera logística, en el sentido de que en casa no se tiene la infraestructura a la altura de muchos trabajos y, por otro lado, que se comparte con la actividad familiar. Así es que los empleados esperan comprensión si deben atender una situación personal y a la vez claridad en lo que se espera de ellos. Muchos sienten más carga de trabajo en la actualidad; con horarios más extendidos, aunque esto probablemente se deba a que tienen que hacer actividades (laborales y hogareñas) a las que no estaban acostumbrados.

Participantes del grupo de empresas grandes estiman que el teletrabajo no es sostenible $100 \%$ del tiempo a largo plazo por las condiciones de riesgo como las ergonómicas y psicosociales. Es posible que se llegue a un 50\% de teletrabajo y 50\% de trabajo en casa, como ya lo están haciendo algunos participantes del estudio.

Quienes ya estaban en la opción de teletrabajo sienten que se debe desmitificar esta modalidad, pues muchas veces trae mayor productividad al mejorar la calidad de vida. Aunque hay individuos que por su personalidad y relaciones familiares no les es posible adaptarse al teletrabajo a largo plazo; caso de los muy dependientes de las relaciones sociales. De todos modos, las reuniones físicas son un polo de desarrollo de ideas difícilmente reemplazables en su totalidad por las opciones tecnológicas actuales; probablemente se puedan suplir un poco con 'telecafés' o algunos otros momentos de distensión. Los trabajadores esperan que los trabajos se orienten más a los resultados que a cumplir un horario.

En general, no se perciben grandes diferencias entre los resultados de ambos países; las preocupaciones y tendencias esperadas son similares. Es posible que la diferencia económica a favor de España se haya diluido en la percepción de los participantes por la crisis que ya se está atravesando, la que se avecina y además, la reciente, que no se presentó en Colombia.

Sin embargo, los participantes colombianos aseguran que temas como aumentar protocolos de limpieza y certificados de bioseguridad ocurrirán después de terminado el confinamiento, mientras que en España sienten que esos temas seguirán igual; esto puede fundamentarse en que en España dichos protocolos estén más avanzados. 
A raíz de la pandemia, hay conciencia de que las acciones de uno afectan a todos. Eso hace que la relación con proveedores sea más fraterna, así como sucede con trabajadores, familiares y vecinos. Las comunicaciones se tornan transparentes y se pueden mostrar los malos momentos por los que se está pasando. La situación a mediano plazo fomentará tener relaciones más duraderas y fuertes con los proveedores y clientes.

Los elementos de bioseguridad pueden afectar el medio ambiente en la medida que se usarán más empaques y protecciones; se harán más desechos. Así es que es importante buscar la manera de protegerse pero con el menor daño posible al medio ambiente.

Sobre mitigar el impacto ambiental, en el grupo de pymes anotaron que lo llevan a cabo en procesos como ahorrar en energía, agua e insumos como papel; que, además de dañar menos el medio ambiente, reflejan costos operativos más bajos.

En cuanto a impacto social, los expertos creen que las organizaciones se centrarán en disminuir costos, también en retener empleo; tienden a considerar que la responsabilidad social se concentrará en los grupos de interés internos como empleados o de afectación directa como clientes más que en relaciones con fundaciones. Además, consideran que se ampliarán las redes sociales y que una de las acciones que pueden darse es apoyar el emprendimiento.

La metodología Delphi permitió tanto estudiar conceptos como buscar pronósticos, como lo explicado por los estudios de Nambisan y Kendall (citados por Okoli \& Pawlowski, 2004).

Con la encuesta de cierre de cada uno de los tres temas en estudio se pudo apreciar que los participantes muestran mayor acuerdo en los puntos sobre las relaciones dentro de la organización; especialmente concuerdan en que se deberán mejorar los procesos de formación para quienes teletrabajen y mejorar los procesos de comunicación interna, pues el error estándar de la media es bajo: 0.097. Es así como las empresas deben evaluar las posibles falencias de sus empleados como el manejo del tiempo en el ámbito del teletrabajo para hacer correctivos en caso de continuar bajo esta modalidad pos pandemia.

Para todos los cambios, es importante formar al equipo comercial para los entornos virtuales, que pueda tener la capacidad de detectar necesidades a distancia. A la vez, que los directores de mercadeo o de recursos humanos logren tener el espíritu de flexibilidad requerido. Se han tenido que tomar decisiones más rápidamente por las circunstancias; se puede aprovechar este aprendizaje pero sin abandonar las herramientas de planeación que se manejaban en el pasado, como fijar grupos objetivos y estrategias de segmentación. Hay un nuevo entorno con nuevas oportunidades y retos; el debatir en tiempo real con colegas de dos países abre el panorama hacia la empatía: a ponerse en el lugar del otro, en su situación. Así es como empresas y consumidores buscan caminos en que sus expectativas se puedan colmar.

\section{Limitaciones}

Algunos participantes opinaron activamente al inicio pero luego no; otros más no lograron vincularse. Además, no todos contestaron los cuestionarios cerrados. Es una muestra pequeña, aunque es lo usual en el tipo de metodología; especialmente el gru- 
po de agremiaciones tuvo un número de participantes reducido. También se anota, que algunos expertos han sido clientes de las empresas organizadoras; otros más se reclutaron por otras vías, como referidos, siempre procurando que se variara el sector económico. Cabe anotar, que por estar en confinamiento, era difícil acceder a números telefónicos empresariales para hacer la invitación a participar en el estudio.

\section{Futuras líneas de investigación}

Se pueden integrar otros países en análisis durante crisis posteriores y hacer comparativos con estudios realizados de forma simultánea con el confinamiento por covid 19, especialmente si se realizaron con otras metodologías. Comprobar en qué medida se han cumplido los pronósticos dentro de un año. En cuanto a metodología, se pueden hacer otros estudios con método Delphi con etapas sincrónicas para fomentar el debate y hacer comparativos con los momentos asincrónicos.

\section{Reconocimientos y Agradecimientos}

La financiación del estudio se hizo con recursos de las dos empresas organizadoras (Infocaribe e Invenies); los participantes actuaron de forma voluntaria.

Se agradece a los participantes del estudio y los otros moderadores: Bernat Rotger y Josep María Caselles.

\section{REFERENCIAS}

Armenakis, A. A. \& Bedeian, A. G. (1999). Organizational Change: A Review of Theory and Research in the 1990s. Journal of Management, 25(3), 293-315. https://doi.org/10.1177/014920639902500303

Barney, J (1991). Firm Resources and Sustained Competitive Advantage. Journal of Management, 17(1), 99-120. https://doi.org/10.1177/014920639101700108

BBC. (2020). Coronavirus: 12 aspectos en los que cambiará radicalmente nuestras vidas. Londres: Redacción British Broadcasting Corporation. Disponible en https://www.bbc.com/mundo/noticias-52512680

Bell, S. J, Auh, A. \& Smalley, K. (2005). Customer Relationship Dynamics: Service Quality and Customer Loyalty in the Context of Varying Levels of Customer Expertise and Switching Costs. Journal of The Academy of Marketing Science, 33(2), 169-183. https://doi.org/10.1177/0092070304269111

Cabanelas, J. Cabanelas, P. y Lorenzo, P. (2007). La gestión de las relaciones con los clientes como característica de la alta rentabilidad empresarial. Revista Europea de Dirección y Economía de la Empresa, 16(3), 133-148. Recuperado de http://agora.edu.es/descarga/articulo/2504842.pdf

Chiaburu, D. \& Harrison, D. (2008). Do peers make the place? Conceptual synthesis and meta-analysis of coworker effects on perceptions, attitudes, OCBs, and performance. Journal of Applied Psychology, 93(5), 1082-1103. https://doi. org/10.1037/0021-9010.93.5.1082 
Cornejo. M. y Tapia, M. (2011). Redes sociales y relaciones interpersonales en Internet. Fundamentos de humanidades, 12(2), 219-229. Disponible en http://fundamentos.unsl.edu.ar/pdf/articulo-24-219.pdf

Coyle-Shapiro, J. \& Kessler, I. (2000). Consequences of the Psychological Contract for the Employment Relationship: A Large-Scale Survey. Journal of Management Studies, 37(7), 903-930. https://doi.org/10.1111/1467-6486.00210

Dalkney, N. \& Helmer, O. (1963). An Experimental Application of the DELPHI Method to the Use of Experts. Management Science, 9(3), 458-467. http://dx.doi. org/10.1287/mnsc.9.3.458

Díaz, C. y Fernández, J. (2015). Marco conceptual de la ética y la responsabilidad social empresarial: un enfoque antropológico y estratégico. Revista Empresa y Humanismo, 19(12), 69-118. https://doi.org/10.15581/015.XIX.2.69-118

Edwards, J. \& Cable, D. (2009). The value of value congruence. Journal of Applied Psychology, 94(3), 654-677. https://doi.org/10.1037/a0014891

García, M. y Forero, C. (2018). Estrés laboral y contrato psicológico como elementos relacionales del cambio organizacional. Perspectiva Psicológica, 14(1), 149-162. Disponible en https://revistas.usantotomas.edu.co/index.php/diversitas/article/ view/4471

Gil-Lafuente, A. y Luis-Bassa, C. (2011). La innovación centrada en el cliente utilizando el modelo de inferencias en una estrategia CRM. Investigaciones Europeas de Dirección y Economía de la Empresa, 17(2), 15-32. https://doi.org/10.1016/ S1135-2523(12)60050-1

Gordon, T. J. (1994). The Delphi Method. Washington, D.C.: The Millennium Project. Disponible en http://www.gerenciamento.ufba.br/downloads/delphi_method. pdf

Gutiérrez, H. y Navallas, B. (2011). La revelación de información social por parte de las empresas españolas: factores explicativos y necesidad de legitimidad social. Contabilidad y Negocios, 6(12), 5-25. Recuperado de http://revistas.pucp.edu. pe/index.php/contabilidadyNegocios/article/view/1644

Humphrey, S., Nahrgang, J. \& Morgeson, F. (2007). Integrating motivational, social, and contextual work design feature: A meta-analytic summary and theorwtical extension of the work design literature. Journal of Applied Psychology, 92(5), 1332-1356. https://doi.org/10.1037/0021-9010.92.5.1332

Kirschner, A. (2006). La responsabilidad social de la empresa. Nueva Sociedad, (202), 133-142. Disponible en https://www.nuso.org/articulo/la-responsabilidadsocial-de-la-empresa/

Landeta, J. (1999). El método Delphi. Una técnica de previsión para la incertidumbre. Barcelona: Ariel.

Leary, T. (1957). Interpersonal diagnosis of personality: A functional theory and methodology for personality evaluation. New York: Ronald Press.

Linstone, H. A. \& Turoff, M. (Eds.) (2002). The Delphi Method. Boston: AddisonWesley Pub.

Lizcano, J. y Moneva, J. (2004). Marco Conceptual para la Responsabilidad Social Corporativa. Madrid: AECA. 
Malott, M. (2001). Paradoja de Cambio Organizacional. Mexico, D.F.: Trillas.

Okoli, C. \& Pawlowski, S. (2004). The Delphi Method as a Research Tool: An Example, Design Considerations and Applications. Information \& Management, 42(1), 15-29. https://doi.org/10.1016/j.im.2003.11.002

Plakoyiannaki, E. \& Saren, M. (2006). Time and the customer relationship management process: conceptual and methodological insights. Journal of Business \& Industrial Marketing, 21(4), 218-230. https://doi.org/10.1108/08858620610672588

Reguant-Álvarez, M. \& Torrado-Fonseca, M. (2016). El método Delphi. REIRE Revista d'Innovació $i$ Recerca en Educació, 9(1), 87-102. https://doi.org/10.1344/ reire2016.9.1916

Rodríguez, L. y Gutiérrez, R. (2019). Estudio prospectivo de escenarios de la tecnología en el trabajo en Colombia al 2050. Económicas CUC, 40(2), 106-116. http://dx.doi.org/10.17981/econcuc.40.2.2019.07

Salas-Arbeláez, L., García-Solarte, M. \& Azuero-Rodríguez, A. R. (2020). Efecto de la responsabilidad social empresarial sobre el posconflicto colombiano: el caso de las pymes. Estudios Gerenciales, 36(154), 80-90. https://doi.org/10.18046/j. estger.2020.154.3547

Santos, J. Tornel, M. \& García, M. (2020). Repercusiones sobre las empresas de sus actuaciones socialmente responsables ante la pandemia generada por el COVID-19. La Razón Histórica, (46), 1-11. Disponible en https://www.revistalarazonhistorica.com/46-1/

Sepúlveda, M. E., Moreno, E. P., Tovar, J. E., Franco, J. A. \& Villarraga, C. L. (2015). Responsabilidad social de las pymes en el marco del posconflicto, como soporte para la inclusión laboral de la población reinsertada. Comunicación, Cultura y Política, 6(1), 45-62. Disponible en https://journal.universidadean. edu.co/index.php/revistai/article/view/1335

Somerville, J. A. (2008). Effective Use of the Delphi Process in Research: Its Characteristics, Strengths and Limitations. [Unpublished doctoral dissertation]. Oregon State University, Corvallis, USA. Available: https://docplayer.net/21500232-Effective-use-of-the-delphi-process-in-research-its-characteristics-strengths-andlimitations-1-jerry-a-somerville.html

Valenzo-Jiménez, M. Martínez-Arroyo, J. Ávila-Carreón, F. (2015). Competitividad en la cadena de suministro y la gestión de relaciones con los clientes en el sector exportador aguacatero. CIMEXUS, 10(1), 71-87. Disponible en https://www. cimexus.umich.mx/index.php/cim1/article/view/206

Vuotto, M. y Arzadun, P. (2007). El buen trabajo y la satisfacción laboral. Documento 59. Buenos Aires: Universidad de Buenos Aires. Disponible en http:// bibliotecadigital-old.econ.uba.ar/download/docin/docin_cesot_059.pdf

Yañez, R., Arenas, M. y Ripoll, M. (2010). El impacto de las relaciones interpersonales en la satisfacción laboral general. Liberabit, 16(2), 193-202. Recuperado de http://ojs3.revistaliberabit.com/publicaciones/revistas/RLE_16_2_el-impactode-las-relaciones-interpersonales-en-la-satisfaccion-laboral-general.pdf 
Zahay, D. \& Griffin, A. (2004). Customer Learning Processes, Strategy Selection, and Performance in Business-to-Business Service Firms. Decision Science, 35(2), 169-203. https://doi.org/10.1111/j.00117315.2004.02338.x

\section{BIODATA}

Marcela Navia-Núñez es psicóloga de la Universidad de la Sabana (Colombia). Cuenta con dos especializaciones de la Universidad del Norte (Colombia): mercadeo y estadística aplicada. Magister en Administración de Empresas de la Universidad del Norte. Gerente de Infocaribe, empresa dedicada a investigación de mercados con sede en Barranquilla (Colombia). Ha sido docente de pregrado y posgrado en áreas de gerencia de mercadeo, investigación de mercados, antropología del consumo y gestión empresarial. ORCID: https://orcid.org/0000-0003-3979-6125

Yanna Stefanu-Lidorikiotu es administradora de empresas de la Escuela de Administración de Empresas de Barcelona (EAE, España), diplomada en educación social de la Universitat Ramon Llul (España). Estudió un posgrado en administración de empresas en CEIPA (Colombia). Es antropóloga social y cultural de la Universidad Nacional de Educación a Distancia (UNED, México). Doctora en Ciencias Sociales y Jurídicas de la Universidad de Cádiz (España). Ha impartido las asignaturas: estrategias de mercadeo en entornos internacionales, plan de mercadeo, investigación de mercados, comunicación corporativa, comunicación interna e interinstitucional, estrategias de desarrollo, lanzamiento de productos y distribución comercial. Profesora de la EAE Business School (Barcelona, España). ORCID: https://orcid.org/00000001-7226-1048 